\title{
Three-dimensional Flow Instability of Natural Convection Induced by Variation in Radius of Inner Circular Cylinder Inside Cubic Enclosure
}

\author{
Young Min Seo, Jeong Hoon Doo and Man Yeong Ha* \\ School of Mechanical Engineering, \\ Pusan National University, \\ 2, Busandaehak-ro 63beon-gil, Busan 46241, South Korea, \\ E-mail: breakfree86@pusan.ac.kr
}

\section{ABSTRACT}

Three-dimensional numerical simulations were conducted for the natural convection phenomena around a hot inner circular cylinder positioned in a cold cubic enclosure in the Raleigh number range of $10^{3} \leq R a \leq 10^{6}$ at the Prandtl number of Pr=0.7. The Immersed Boundary Method (IBM) was used to capture the virtual wall boundary of the inner cylinder, based on the Finite Volume Method (FVM). In this study, the transition of the flow regime from the steady state to the unsteady state and consequent three-dimensionality in the system induced by the increase in the flow instability were investigated. Detailed three-dimensional vortical structures of the convection cells at a relatively high Rayleigh number of $R a=10^{6}$ were analysed using the visualization technique, and the heat transfer characteristics in the system resulting from the change in the vortical structures were addressed.

\section{INTRODUCTION}

Natural convection in an enclosure has various applications, ranging from industrial fields to environmental fields, such as heat exchangers, nuclear reactors safety technologies, electronics cooling to building thermal design, solar energy systems and stratified atmospheric boundary layers. The thermo-fluid behavior of those systems also entrains the fundamental knowledge of flow stability. More generally, almost all technologies involving passive heat transfer as the main source of thermal dissipation rely upon natural convection effects. For this reason, the relevant literatures have focused on heat transfer performances [1-2]. Natural convection heat transfer exhibits a great variety of complex dynamic behaviors, which depend greatly on the geometry and thermal conditions of the enclosure. Thus, the theoretical study of the phenomenon is comparatively difficult to understand and the experimental analysis of such systems has always inherent difficulties [3-4].

For several decades many researchers have investigated the effects of various parameters on natural convection in an enclosure with an inner body as the numerical methodology was developed. The horizontal concentric cylinder geometry inside an enclosure is used in pressurized-gas underground electric transmission cables [5]. Additionally, the annulus geometry has application in solar collector receiver and thermal storage systems [6]. According to the results of these previous studies, natural convection in an enclosure depends on the size and position of an inner cylinder [7-10].

\section{NOMENCLATURE}

\begin{tabular}{|c|c|}
\hline \multicolumn{2}{|c|}{ Symbols } \\
\hline$A$ & Surface area of cylinder and walls of enclosure. \\
\hline$f_{i}$ & Momentum forcing \\
\hline$g$ & Gravitational acceleration \\
\hline$H$ & Vertical length of enclosure \\
\hline$h$ & heat source or sink \\
\hline$L$ & Edge length of enclosure \\
\hline$n$ & Direction normal to the wall \\
\hline $\mathrm{Nu}$ & Nusselt number \\
\hline$P$ & Dimensionless pressure \\
\hline $\operatorname{Pr}$ & Prandtl number \\
\hline$q$ & Mass source or sink \\
\hline$R$ & Radius of circular cylinder \\
\hline$R a$ & Rayleigh number \\
\hline$T$ & Temperature \\
\hline$t$ & Dimensionless time \\
\hline$u_{i}$ & Dimensionless velocity vector \\
\hline$x_{i}$ & Cartesian coordinates system \\
\hline \multicolumn{2}{|c|}{ Greek symbols } \\
\hline$\alpha$ & Thermal diffusivity \\
\hline$\beta$ & Thermal expansion coefficient \\
\hline$\theta$ & Dimensionless temperature \\
\hline$\lambda_{2}$ & Lamda- 2 criteria for vortical structure \\
\hline$v$ & Kinematic viscosity \\
\hline$\rho$ & Density \\
\hline$\tau_{p}$ & Period of the fluctuation of thermal and flow fields \\
\hline$\Omega$ & Volume of fluid \\
\hline \multicolumn{2}{|c|}{ Sub/superscripts } \\
\hline$B$ & Bottom wall \\
\hline$c$ & Cold \\
\hline$C$ & Cylinder \\
\hline$E N$ & Enclosure \\
\hline$i, j$ & Tensor notation \\
\hline$H$ & Hot \\
\hline$T$ & Top wall \\
\hline$*$ & Dimensional variable \\
\hline
\end{tabular}

Moukalled and Acharya [7] studied the natural convection heat transfer between a heated horizontal cylinder placed concentrically inside a square enclosure. Three different diameter-to-side aspect ratios between 0.1 and 0.3 were considered. The range of the Rayleigh number is $10^{4} \leq R a \leq 10^{7}$. According to their research, the total heat transfer increases with increasing Rayleigh number at constant enclosure aspect ratio. At constant Rayleigh numbers, the convection 
contribution to the total heat transfer decreases with increasing values of aspect ratios.

Shu and Zhu [8] studied the variation in the thermal and flow fields in a square enclosure with respect to the various radii of an inner circular cylinder. They obtained the results in the Rayleigh number range of $10^{4} \leq R a \leq 10^{6}$ with the Prandtl number of 0.71 and the diameter-to-side aspect ratios between 1.67 and 5.0. It was found that both the diameter-to-side aspect ratio and the Rayleigh number are critical to the patterns of thermal and flow fields in the enclosure.

Angeli et al. [9] investigated the effect of the radius of an inner cylinder on the heat transfer between the cylinder and the square enclosure. The range of the Rayleigh number is $10^{2} \leq R a \leq 5 \times 10^{6}$, and the Prandtl number is 0.7 . Four values of the diameter-to-side ratios were considered: $0.2,0.4,0.6$, and 0.8 . According to their research, stable symmetric and nonsymmetric steady-state solutions as well as unsteady regimes are observed, depending on the Rayleigh number and the aspect ratio of the cavity.

Kim et al. [10] studied on the natural convection in the square enclosure with respect to the position of the inner circular cylinder. The range of the Rayleigh number is $10^{3} \leq R a \leq 10^{6}$, and the Prandtl number is 0.7 . The inner circular cylinder moved along the vertical centerline of the enclosure in the range of $-0.25 \leq \delta \leq 0.25$. They reported that the number, size and formation of convection cells in the enclosure depend on the location of the inner cylinder as well as the Rayleigh number.

In this study, therefore, authors consider three-dimensional configuration of the system having a cubic enclosure and an inner circular cylinder in order to investigate the transition of the flow regime from the steady state to the unsteady state due to the flow instability in the Rayleigh number range of $10^{3} \leq R a \leq 10^{6}$ when the radius of the inner circular cylinder varies in $0.1 \mathrm{~L} \leq R \leq 0.4 \mathrm{~L}$ as well as the consequent threedimensionality in the system. Detailed three-dimensional vortical structures of the convection cells at a relatively high Rayleigh number of $R a=10^{6}$ were analyzed using the visualization technique, and the heat transfer characteristics in the system resulting from the change in the vortical structures were addressed.

\section{NUMERICAL METHODS}

In this study, the immersed boundary method based on the finite volume method is used to capture the virtual wall boundary of an inner circular cylinder positioned in the cubic enclosure. The immersed boundary method has a benefit to handle more easily an inner object having a complex geometry in the Cartesian coordinate system. Further details on the immersed boundary method are described in Kim et al. [11] and Kim and Choi [12].

The governing equations for mass, momentum, and energy conservation using the immersed boundary method are defined in their non-dimensional forms as follows:

$$
\frac{\partial u_{i}}{\partial x_{i}}-q=0
$$

$$
\begin{aligned}
& \frac{\partial u_{i}}{\partial t}+u_{j} \frac{\partial u_{i}}{\partial x_{j}}=-\frac{\partial P}{\partial x_{i}}+\operatorname{Pr} \frac{\partial^{2} u_{i}}{\partial x_{j} \partial x_{j}}+\operatorname{RaPr} \theta \delta_{i 2}+f_{i} \\
& \frac{\partial \theta}{\partial t}+u_{j} \frac{\partial \theta}{\partial x_{j}}=\frac{\partial^{2} \theta}{\partial x_{j} \partial x_{j}}+h
\end{aligned}
$$

The dimensionless variables in the above equations are defined as:

$t=\frac{t^{*} \alpha}{L^{2}}, x_{i}=\frac{x_{i}^{*}}{L}, u_{i}=\frac{u_{i}^{*} L}{\alpha}, P=\frac{P^{*} L^{2}}{\rho \alpha^{2}}, \theta=\frac{T^{*}-T_{c}^{*}}{T_{h}^{*}-T_{c}^{*}}$

In Eq. (4), $\rho, T$, and $\alpha$ represent the density, dimensional temperature, and thermal diffusivity, respectively. The superscript (*) in Eq. (4) represents the dimensional variables; $x_{i}$ represents Cartesian coordinates, $u_{\mathrm{i}}$ the corresponding velocity components, $t$ the time, $p$ the pressure and $\theta$ the temperature. The fluid properties are assumed to be constant except the density in the buoyancy term, which follows the Boussinesq approximation.

The preceding non-dimensionalization produces two important dimensionless parameters: $\operatorname{Pr}=v / \alpha$ and $\operatorname{Ra}=g \beta L^{3}\left(T_{h}{ }^{*}{ }^{-}\right.$ $\left.T_{h}{ }^{*}\right) / v \alpha$, where $v, g$, and $\beta$ are the kinematic viscosity, gravitational acceleration, and volume expansion coefficient, respectively. The terms of $q, f_{i}$, and $h$ in Eqs. (1)-(3) are extra terms in the governing equations to which the immersed boundary method is applied. The mass source/sink $q$, in Eq. (1) and momentum force $f_{i}$, in Eq. (2) were applied to the surface or inside the body to satisfy the no-slip condition and mass conservation in the cell containing the immersed boundary. In Eq. (3), the heat source/sink $h$, was applied to satisfy the isothermal boundary condition on the surface of the inner body. A second-order linear or bilinear interpolation scheme was applied to satisfy the no-slip and isothermal conditions on the immersed boundary.

The central difference scheme with second-order accuracy based on the finite-volume method was used for spatial discretization. In the discretization process, the advection terms were explicitly treated by using the second-order AdamsBashforth scheme while the diffusion terms were implicitly treated by using the second-order accurate Crank-Nicolson scheme.

The local and surface-averaged Nusselt numbers, $N u$ and $\langle N u\rangle$, are defined, respectively, as

$$
N u=\left.\frac{\partial \theta}{\partial n}\right|_{\text {wall }}, \quad\langle N u\rangle=\frac{1}{A} \int N u d A
$$

where $n$ is the normal direction with respect to the wall and $A$ is the surface area of the cylinder and walls of the enclosure.

\section{COMPUTATIONAL CONDITIONS}

Figure 1 shows the computational domain of the cubic enclosure and its coordinate system considered in this study. The inner circular cylinder is located at the center of the enclosure. In this figure, $L$ and $R$ represent the edge length of the enclosure and the radius of the inner cylinder, respectively. In this study, the radius of the inner circular cylinder as a main simulation parameter is considered to be $R=0.1 L, R=0.2 L$, $R=0.3 L$ and $R=0.4 L$. The Rayleigh number range considered in 
this study is $10^{3} \leq R a \leq 10^{6}$, and the Prandtl number is set to be $\operatorname{Pr}=0.7$ for all numerical simulations. No-slip and impermeability boundary conditions are imposed on the walls of the enclosure and the surface of the inner cylinder. The adiabatic condition is imposed on the front and rear walls of the cubic enclosure. The other walls of the cubic enclosure are kept at a constant low temperature $\theta_{c}=0$. The surface of the inner circular cylinder is kept a constant high temperature $\theta_{h}=1$. The gravitational acceleration acts in the negative $y$-direction.

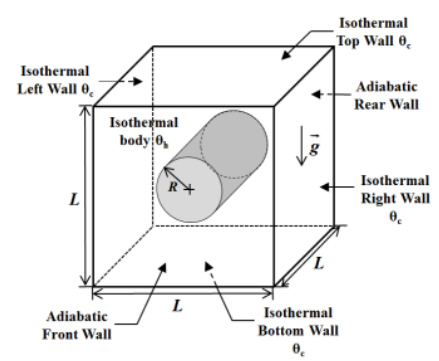

Figure 1 Computational domain and coordinate system along with boundary conditions.

Very dense grids with the uniform distribution in the $x$ and $y$ directions are generated in the section where the inner circular cylinder is located in order to capture the virtual wall boundary of the inner circular cylinder accurately when the immersed boundary method is used. In the other section, non-uniform grids controlled by the hyperbolic tangent function are distributed to minimize the computational cost as well as improve the resolution for high velocity and temperature gradients near walls of the enclosure. The minimum size of the grid spacing is $L / 250$ and the maximum size $3 L / 200$. The grid dependency test was conducted for much denser grids with the minimum mesh size of $L / 350$. The test results show that the numerical solutions are almost independent on the increase in the number of grid points. The discrepancy between result in the current grid system and that in a denser grid system is less than $0.3 \%$ in terms of the surface-averaged Nusselt number on the cylinder surface.

\section{VALIDATION TEST}

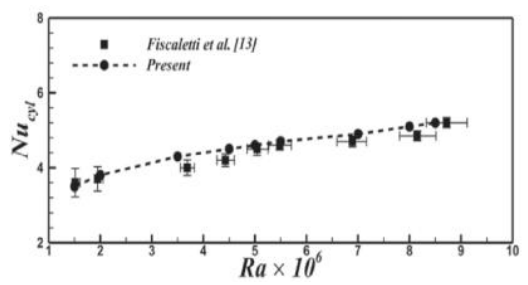

Figure 2 Results of validation test with respect to the Rayleigh number: Surface-averaged Nusselt number of the inner circular cylinder

The preliminary numerical simulations on the natural convection problem considered by Fiscaletti et al. [13] were conducted to validate the present numerical simulation code. And results obtained by the present code were compared to those obtained by Fiscaletti et al. [13]. Figure 2 shows the surface-averaged Nusselt number on the cylinder surface as a function of the Rayleigh number in the range of $1.5 \times 10^{6} \leq R a \leq 8 \times 10^{6}$. In this figure, the surface-averaged Nusselt numbers $N u_{c}$ on the cylinder surface of the present study match well with those of previous study. As shown in this figure, excellent agreements with previous study were achieved.

\section{RESULTS AND DISCUSSION}

In this study, the Rayleigh number of $10^{3} \leq R a \leq 10^{6}$ and the radius of the inner circular cylinder of $0.1 L \leq R \leq 0.4 L$ are considered. At a relatively low Rayleigh numbers of $R a=10^{3}$, $R a=10^{4}$ and $R a=10^{5}$, the numerical solutions show the timeindependent characteristics regardless of the variation in the radius of the inner cylinder. In addition, the flow and thermal structures obtained in three-dimensional domain configuration reveals almost two-dimensional pattern in the spanwise direction (z), and three-dimensionality in the system is not observed in this Rayleigh number range as shown in figures 3(a) $\sim 3(\mathrm{c})$.

When the Rayleigh number increases to $R a=10^{6}$, numerical solutions at $R=0.1 L$ and $R=0.2 L$ still maintain a steady state. However, when the radius of the inner cylinder increases further to $R=0.3 L$ and $R=0.4 L$, the flow experiences the transition from the steady state to the unsteady state, and threedimensional vortical structures of the convection cell occur in the enclosure. In this paper, therefore, authors focus on the flow instability and the consequent flow transition from the steady state to the unsteady state when the radius of the inner cylinder varies at a relatively high Rayleigh number of $R a=10^{6}$. In addition, the dynamic characteristics of three-dimensional vortical structures according to time evolution are highlighted in this paper.
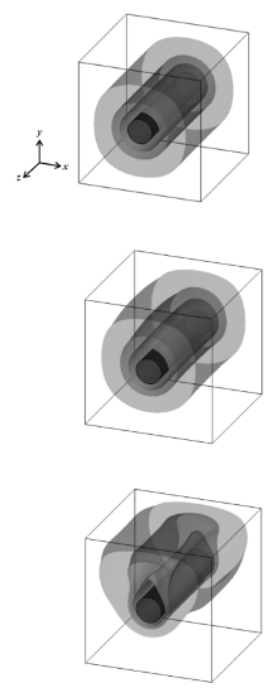

(a)
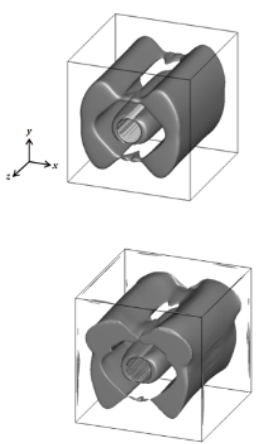

(b)

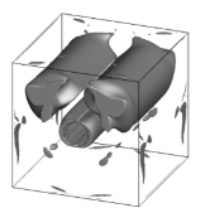

(c)

Figure 3 Isothermals (3D view) and vortical structure (3D view) at $R=0.1 L$; (a) $R a=10^{3}$, (b) $R a=10^{4}$ and (c) $R a=10^{5}$.

In this study, three-dimensional vortical structures are visualized using the method proposed by Jeong and Hussain [14]. A negative $\lambda_{2}$ close to zero is recommended to reveal the 
most outer surface of the vortical structures according to their research, where $\lambda_{2}$ denotes the second largest eigenvalue of $S^{2}+\Omega^{2}$, where $S^{2}$ and $\Omega^{2}$ are the strain and rotation rates of the velocity gradient tensor $\nabla u_{\mathrm{i}}$. However, the iso-surface value of $\lambda_{2}=-0.1$ is adopted in the visualization of the vortical structures in order to clearly decipher the vortical structures distributed in the enclosure.

Figure 4(a) shows the time history of the surface-averaged Nusselt number on the top wall of the enclosure $\left\langle N u_{T}\right\rangle$ at $R=0.3 L$ when $R a=10^{6}$ and figure 4(b) its power spectra as a function of the frequency. Figures $4(\mathrm{c}) \sim 4(\mathrm{f})$ show the time evolution of three-dimensional vortical structures formed in the upper part of the cylinder at four instants corresponding each time $(t=A \sim D)$ specified in figure 4(a). Here, the rotational direction of the vortical structure is defined by the right-hand rule about its rotation axis and specified in these figures. When the positive $x$-direction is the rotation axis, the clockwise (CW) direction indicates the rotation in accordance with the righthand rule, and the counter-clockwise (CCW) direction indicates its inverse rotation direction.

In figure 4(a), the time history $\left\langle N u_{T}\right\rangle$ shows the unsteady characteristics, and its power spectrum has the primary frequency of approximately 56 with several harmonics at lower and higher frequencies than the primary one in a wide frequency band of $0<f<200$ as shown in figure 4(b). Therefore, the oscillating motion of $\left\langle N u_{T}\right\rangle$ as function of time is substantially modulated from a fundamental sinusoidal wave at the primary frequency.

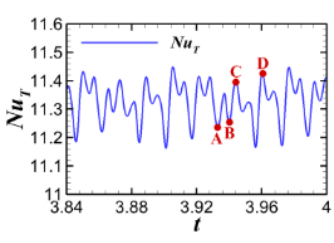

(a)

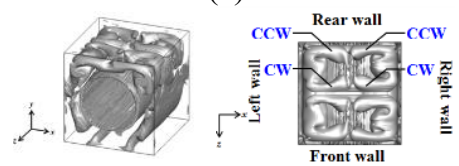

(c)
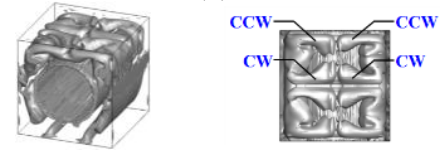

(e)

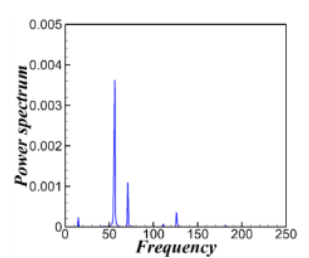

(b)
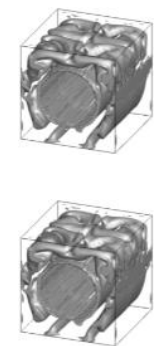

(d)
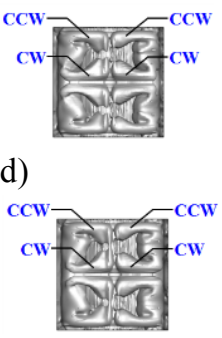

(f)

Figure $4 R=0.3 L$ at $R a=10^{6}$; (a) time history of $\mathrm{Nu}$ on the top wall of the enclosure, (b) time evolution of the convection cell (3D view and top view).

As shown in figure 4(a), the variation in $\left\langle N u_{T}\right\rangle$ according to time is not big, and the maximum amplitude is within approximately $\pm 1.4 \%$ about the time-averaged value. Therefore, three-dimensional vortical structures formed in the upper part of the cylinder keep their shapes almost stable as time goes on as shown in figures $4(\mathrm{c}) \sim 4(\mathrm{f})$. When the radius of the cylinder becomes increased from $R=0.2 L$ to $R=0.3 L$, the space that the spanwise vortex occupies is confined and hence the flow instability is intensified. As a result, a high kinetic energy of the spanwise vortex is transferred to the spanwise velocity component, which leads to the occurrence of the streamwise vortex. In this process, the angular momentum of the spanwise vortex is redistributed to the streamwise vortex, satisfying the conservation of the angular momentum. As shown in figures 4(c) $\sim 4(f)$, four different streamwise vortices are generated near the mid-plane normal to the $\mathrm{z}$ direction and two streamwise vortices near each lateral wall (front and rear walls). In contrast to the two-dimensional convection structure in the cases of $R=0.1 L$ and $R=0.2 L$, the hot or cold fluid is transported upward or downward, following the circulation motion of the streamwise vortices at $R=0.3 \mathrm{~L}$, which leads to the distortion of the isothermals near the top wall of the enclosure along the spanwise direction.

In figure 5(a), the time history of $\left\langle N u_{T}\right\rangle$ shows the unsteady characteristics, and its power spectrum has the primary frequency of approximately 4.5 with several harmonics at frequencies that are very close to the primary frequency in $0<f<25$ as shown in figure 5(b). As a result, the periodicity in the signal of $\left\langle N u_{T}\right\rangle$ is more clearly identified, compared to the case of $R=0.3 L$. Similar to the case of $R=0.3 L$, the amplitude of $\left\langle N u_{T}\right\rangle$ is still small within $\pm 1.7 \%$ about the time-averaged value in this case. However, in contrast to the case of $R=0.3 L$, the change in three-dimensional vortical structure according to time is clearly identified in figures 5(c) $\sim 5(\mathrm{f})$.

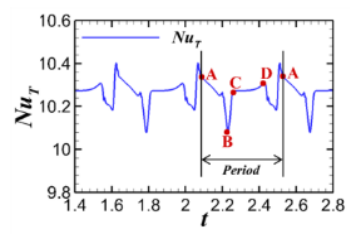

(a)

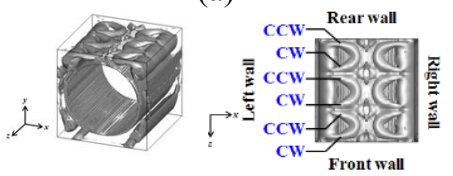

(c)
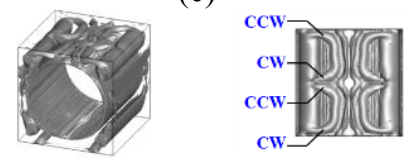

(e)

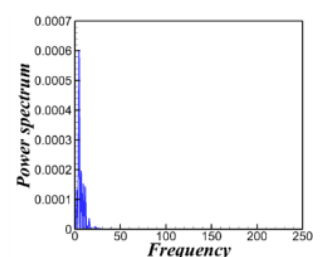

(b)
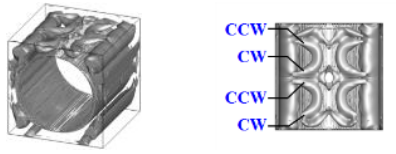

(d)
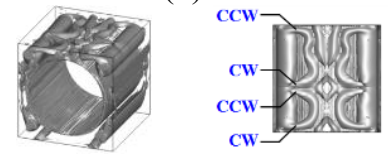

(f)
Figure $5 R=0.4 L$ at $R a=10^{6}$; (a) time history of $\mathrm{Nu}$ on the top wall of the enclosure, (b) time evolution of the convection cell (3D view and top view).

When the radius of the cylinder increases further to $R=0.4 \mathrm{~L}$ from $R=0.3 \mathrm{~L}$, the space in which the fluid is filled is extremely confined. As a result, the circulating motion of the fluid in the enclosure is suppressed, and hence the magnitude of the streamwise vortex decreases, compared to the case of $\mathrm{R}=0.3 \mathrm{~L}$. In figure $5(\mathrm{c})$, at $\mathrm{t}=\mathrm{A}$, it is observed that the $\mathrm{C}$-shaped vortical 
structure is generated by the combination of a pair of two streamwise vortices and a single spanwise vortex, and the triple C-shaped vortical structures are located in the left and right sides of the cylinder separately with almost the same spanwise period of $\mathrm{L} / 3$. When $\mathrm{t}=\mathrm{B}$ as shown in figure 5(d), two $\mathrm{C}$-shaped vortical structures, which are located near the mid-plane normal to the $\mathrm{z}$ direction and front wall of the enclosure, become increases in their spanwise size, and hence the other C-shaped vortical structure located near the rear wall of the enclosure is confined. When $\mathrm{t}=\mathrm{C}$ as shown in figure $5(\mathrm{e})$, the triple vortical structures with the spanwise period of L/3 is changed to the bilingual vortical structure with the spanwise period of $\mathrm{L} / 2$. As time goes on further to $\mathrm{t}=\mathrm{D}$ as shown in figures $5(\mathrm{f})$, the $\mathrm{C}$ shaped vortical structure located near the front wall of the enclosure is getting decreased in its spanwise length, and the other one located near the rear wall of the enclosure initiates to be separated into two parts. Finally, the bilingual vortical structure with the spanwise period of $\mathrm{L} / 2$ is returned back to the triple vortical structure with the spanwise period of $L / 3$ as shown in figure 5(c). These vortical structures show a mirrorsymmetric pattern about the mid-plane normal to the $\mathrm{x}$ direction at each instant.
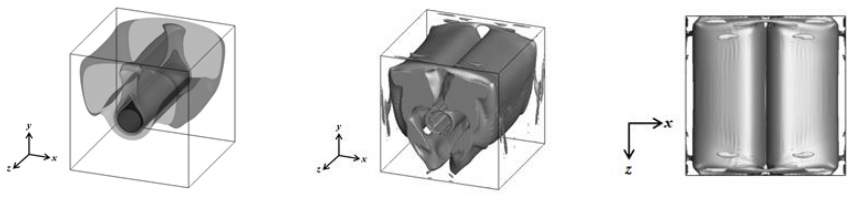

(a)
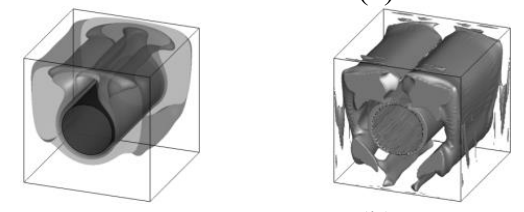

(b)
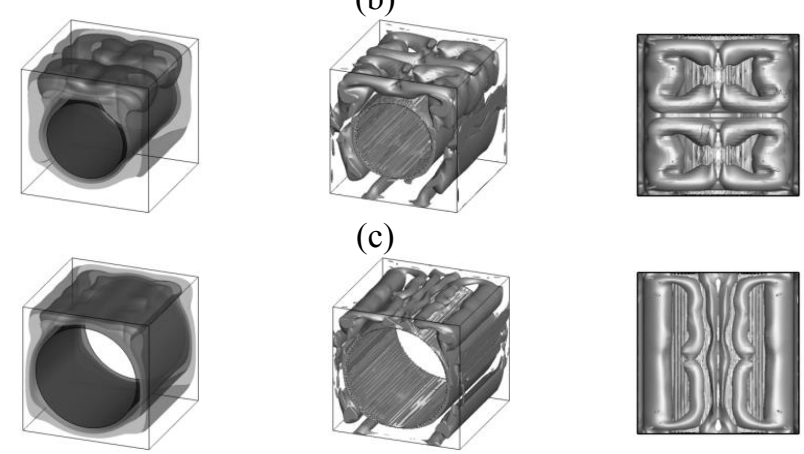

(c)
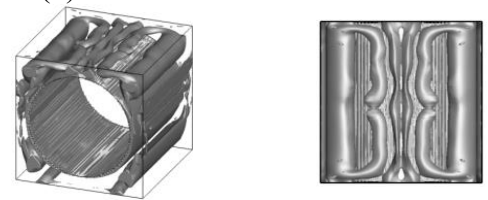

(d)

Figure 6 Isothermals (3D), vortical structure (3D), vortical structure (top view) at $R a=10^{6}$; (a) $R=0.1 L$, (b) $R=0.2 L$, (c) $R=0.3 L$, (d) $R=0.4 L$.

Figures 6(a) $\sim 6($ d) show three-dimensional vortical structures and isothermals in the enclosure at $R=0.1 L, R=0.2 L$, $R=0.3 L$ and $R=0.4 L$ when $R a=10^{6}$, respectively. Here, figures 6(a) and 6(b) are the results obtained from the steady state numerical solutions and figures 6(c) and 6(d) the results obtained from the time-averaged flow and thermal fields.
As aforementioned, the vortical structures in the enclosure at $R=0.1 L$ and $R=0.2 L$ show almost two-dimensional patterns, but the roll-up motions of the spanwise vortex are identified in the near-wall region of each lateral wall of the enclosure (i.e. front and rear walls) at $R=0.1 L$ and $R=0.2 L$ as shown in figures $6(\mathrm{a})$ and $6(\mathrm{~b})$, and the motions are generated by the no-slip condition on the lateral walls of the enclosure. As the radius of the cylinder increases from $R=0.1 L$ to $R=0.2 L$, the influence of the ascending plume on the top wall is intensified and spreads farther to the left and right walls due to a shorter distance between the cylinder surface and top wall.

When the radius of the cylinder increases to $R=0.3 L$, threedimensional vortical structures having the streamwise vortices are formed in the time-averaged flow field. As expected from the unsteady characteristics of the vortical structures presented in figure 4(c) 4(f) showing a very small variation in their structure according to time, the vortical structures in the timeaveraged flow field are very similar to those in the instantaneous flow field. The structure of the ascending plume above the cylinder surface is substantially distorted by the fluid motion of the streamwise vortices along the spanwise direction.

When the radius of the cylinder increases further to $R=0.4 L$, the convection flow in the enclosure is extremely confined in the upper-left, upper-right, lower-left and lower-right parts of the cylinder with a very small space, and the fluid motion of the spanwise and streamwise vortices is very limited. As a result, the streamwise vortical structures presented in figure 5(c) $\sim 5(\mathrm{f})$ almost vanish in the time-averaged flow field, and a pair of the streamwise vortices only is formed near each lateral wall of the enclosure. Due to this, the isothermals of the ascending plume above the cylinder surface is distorted slightly along the spanwise direction near the left and right walls of the enclosure but is quite uniform along the spanwise direction near the midplane normal to the $\mathrm{x}$ direction.

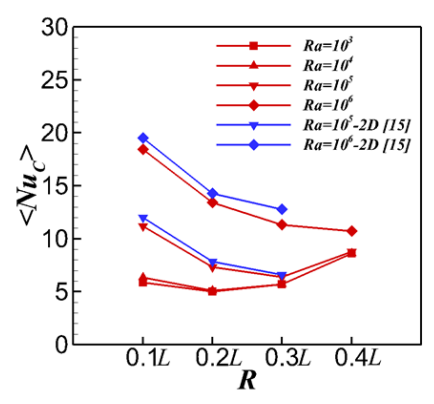

(a)

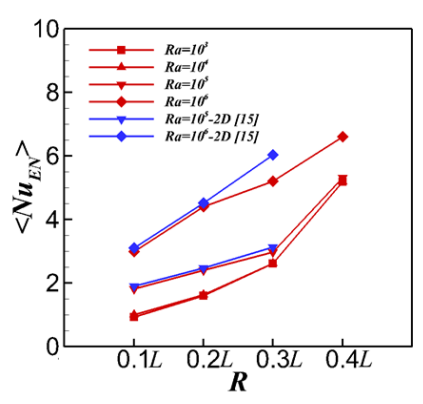

(b)
Figure 7 Surface-averaged $N u$ distribution against $R$ variation at $R a=10^{3}, R a=10^{4}, R a=10^{5}$ and $R a=10^{6}$; (a) cylinder surface and (b) walls of the enclosure.

In figure $7(\mathrm{a})$, a very small difference in $\left\langle N u_{\mathrm{c}}\right\rangle$ between three-dimensional results and two- dimensional ones is identified at $R a=10^{5}$ in the $0.1 L \leq R \leq 0.3 L$, and three-dimensional results are slightly lower than two-dimensional ones due to the presence of the lateral walls of the enclosure (i.e. front and rear walls) in the three-dimensional enclosure configuration. Such a feature is also identified as shown in figure 7(b). When $R a=10^{6}$, 
the difference in $\left\langle N u_{\mathrm{c}}\right\rangle$ between three-dimensional results and two-dimensional results increases as the radius of the cylinder increases from $R=0.1 L$ and $R=0.2 L$ to $R=0.3 L$ as shown in figure $7(a)$. And such a feature is more clearly observed in figure 7(b). The difference between three-dimensional results and two-dimensional ones at $R=0.3 L$ results from the transition of the flow regime from the steady state to unsteady state, and the flow and thermal structures are altered from twodimensional structure to three-dimensional one.

In figure 7(a), the values of $\left\langle N u_{\mathrm{c}}\right\rangle$ decrease from $R=0.1 L$ to $R=0.2 L$ and then increase from $R=0.2 L$ to $R=0.4 L$ at $R a=10^{3}$ and $R a=10^{4}$. The values of $\left\langle N u_{\mathrm{c}}\right\rangle$ decrease from $R=0.1 L$ to $R=0.3 L$ and then increase from $R=0.3 L$ to $R=0.4 L$ at $R a=10^{5}$. When $R a=10^{6}$, the values of $\left\langle N u_{\mathrm{c}}\right\rangle$ decrease monotonically from $R=0.1 L$ to $R=0.4 L$. In figure $12(\mathrm{~b})$, the values of $\left\langle N u_{\mathrm{c}}\right\rangle$ increase monotonically with increasing the radius of the cylinder in the whole Rayleigh number range considered in this study.

\section{CONCLUSION}

In this study, three-dimensional numerical analysis was conducted on the natural convection problem defined in the cubic enclosure having an inner circular cylinder. As a key simulation parameter, the Rayleigh number of $10^{3} \leq R a \leq 10^{6}$ and the radius of the cylinder of $0.1 \mathrm{~L} \leq R \leq 0.4 \mathrm{~L}$ were considered.

In the Rayleigh number range of $10^{3} \leq R a \leq 10^{5}$, the numerical solutions show the time-independent characteristics, and the flow and thermal structures have almost a two-dimensional structure regardless of the variation of the radius of the cylinder. When the Rayleigh number increases to $R a=10^{6}$, the numerical solutions still show the time-independent characteristics, and the flow and thermal structure have almost two-dimensional structure at $R=0.1 L$ and $R=0.2 L$. However, as the radius of the cylinder increases further to $R=0.3 L$ at $R a=10^{6}$, the flow experiences a transition from the steady state to unsteady state. When the radius of the cylinder increases further to $R=0.4 L$, the flow and thermal structures still show the unsteady characteristics.

When the radius of the cylinder increases from $R=0.2 L$ to $R=0.3 L$ at $R a=10^{6}$, the space that the spanwise vortex occupies is confined and hence the flow instability is intensified. As a result, a high kinetic energy of the spanwise vortex is transferred to the spanwise velocity component, which leads to the occurrence of the streamwise vortex. In this process, the angular momentum of the spanwise vortex is redistributed to the streamwise vortex, satisfying the conservation of the angular momentum.

When the radius of the cylinder increases further to $R=0.4 \mathrm{~L}$ from $R=0.3 L$ at $R a=10^{6}$, the space in which the fluid is filled is extremely confined. As a result, the circulating motion of the fluid in the enclosure is suppressed, and hence the magnitude of the streamwise vortex decreases, compared to the case of $R=0.3 L$.

\section{ACKNOLEDGEMENTS}

This work was supported by the National Research Foundation of Korea (NRF) grant funded by the Korea government (MSIP) (No. NRF-2013R1A2A2A01067251).

\section{REFERENCES}

[1] D. Angeli, A. Pagano, M.A. Corticelli, A. Fichera, G.S. Barozzi, Bifucations of Natural Convection Flows from an Enclosed Cylindrical Heat Source, Frontiers in Heat and Mass Transfer 2 (2011) 023003.

[2] X. Xu, Z. Yu, Y. Hu, L. Fan, K. Cen, A Numerical Study of laminar Natural Convective Heat Transfer around a Horizontal Cylinder inside a Concentric Air-Filled Triangular Enclosure, International Journal of Heat and mass Transfer 53 (2010) 345-355.

[3] F. Corvaro, M. Paroncini, An Experimental Study of Natural Convection in a Differentially Heated Cavity through a 2D-PIV System, International Journal of Heat and Mass Transfer 52 (2009) 335-365.

[4] C. Butler, D. Newport, M. Geron, Natural Convection Experiments on a Heated Horizontal Cylinder in a Differentially Heated Square Cavity, Experimental Thermal and Fluid Science 44 (2013) 199-208.

[5] T. H. Kuehn, R. J. Goldstein, An Experimental and Theoretical Study of Natural Convection in the Annulus between Horizontal Concentric Cylinders, Journal of Fluid Mechanics 74 (1976) 695 719.

[6] R. Kumar, Study of natural convection in horizontal annuli, International Journal of Heat and Mass Transfer 3 (1988) 1137-1148.

[7] F. Moukalled, S. Acharya, Natural Convection in the Annulus Between Concentric Horizontal Circular and Square Cylinders, Journal of Thermophysics and Heat Transfer 10 (1996) 524-531.

[8] C. Shu, Y. D. Zhu, Efficient Computation of Natural Convection in a Concentric Annulus between an Outer Square Cylinder and an Inner Circular Cylinder, International Journal for Numerical Methods in Fluids 38 (2002) 429-445.

[9] D. Angeli, P. Levoni, G.S. Barozzi, Numerical Predictions for Stable Buoyant Regimes within a Square Cavity Containing a Heated Horizontal Cylinder, International Journal of Heat and Mass Transfer 51 (2008) 553-565.

[10] B.S. Kim, D.S. Lee, M.Y. Ha, H.S. Yoon, A Numerical Study of Natural Convection in a Sqaure Enclosure with a Circular Cylinder at Different Vertical Locations, International Journal of Heat and Mass Transfer 51 (2008) 1888-1906.

[11] J. Kim, D. Kim, H. Choi, An Immersed-Boundary Finite Volume Method for Simulations of Flow in Complex Geometries, Journal of Computational Physics 171 (2001) 132-150.

[12] J. Kim, H. Choi, An Immersed-Boundary Finite-Volume Method for Simulation of Heat Transfer in Complex Geometries, KSME International Journal 18 (2004) 1026-1035.

[13] D. Fiscaletti, D. Angeli, L. Tarozzi, G.S. Barozzi, BuoyancyInduced Transitional Flows around an Enclosed Horizontal Cylinder: An Experiment, International Journal of Heat and Mass Transfer 58 (2013) 619-631.

[14] J. Jeong, F. Hussain, On the identification of a vortex, J. Fluid Mech. 285 (1995) 69-94

[15] H.K. Park, M.Y. Ha, H.S. Yoon, Y.G. Park, Changmin Son, A numerical study on natural convection in an inclined square enclosure with a circular cylinder, International Journal of Heat and Mass Transfer, 66 (2013) 295-314. 